\title{
Effect of Plant Polyphenols on Adipokine Secretion from Human SGBS Adipocytes
}

\author{
Christos S. Derdemezis, ${ }^{1}$ Dimitrios N. Kiortsis, ${ }^{1}$ Vasilis Tsimihodimos, ${ }^{2}$ \\ Maria P. Petraki, ${ }^{3}$ Patra Vezyraki, ${ }^{1}$ Moses S. Elisaf, ${ }^{2}$ and Alexandros D. Tselepis ${ }^{3}$ \\ ${ }^{1}$ Laboratory of Physiology, School of Medicine, University of Ioannina, 45110 Ioannina, Greece \\ ${ }^{2}$ Department of Internal Medicine, School of Medicine, University of Ioannina, 45110 Ioannina, Greece \\ ${ }^{3}$ Department of Chemistry, University of Ioannina, 45110 Ioannina, Greece
}

Correspondence should be addressed to Alexandros D. Tselepis, atselep@uoi.gr

Received 19 April 2011; Revised 4 July 2011; Accepted 23 July 2011

Academic Editor: Tzi-Bunb Ng

Copyright (C) 2011 Christos S. Derdemezis et al. This is an open access article distributed under the Creative Commons Attribution License, which permits unrestricted use, distribution, and reproduction in any medium, provided the original work is properly cited.

Introduction. Adipose tissue contributes to atherosclerosis with mechanisms related to adipokine secretion. Polyphenols may exhibit antiatherogenic properties. The aim of the study was to investigate the effects of three polyphenols, namely, quercetin, epigallocatechin gallate (EGCG), and resveratrol on adipokine secretion from cultured human adipocytes. Methods. Human SGBS adipocytes were treated with quercetin, EGCG, and resveratrol for 24 and 48 hours. Visfatin, leptin, and adiponectin were measured in the supernatant. Results. Visfatin secretion was inhibited by quercetin $10 \mu \mathrm{M}$ by $16 \%$ and $24 \%$ at 24 and 48 hours respectively. The corresponding changes for quercetin $25 \mu \mathrm{M}$ were $47 \%$ and $48 \%$. Resveratrol $25 \mu \mathrm{M}$ reduced visfatin by $28 \%$ and $38 \%$ at 24 and 48 hours. EGCG did not have an effect on visfatin. None of tested polyphenols influenced leptin and adiponectin secretion. Conclusion. Quercetin and resveratrol significantly decreased visfatin secretion from SGBS adipocytes. This effect may contribute to their overall antiatherogenic properties.

\section{Introduction}

Obesity, defined as body mass index over $30 \mathrm{~kg} / \mathrm{m}^{2}$, has emerged over the past thirty years as a major public health issue. The extent of the problem is ever-growing with incidence reaching epidemic dimensions, especially in Western societies. Obesity increases the risk of developing insulin resistance, diabetes mellitus, hypertension, and coronary heart disease and overall is associated with elevated morbidity and mortality [1]. Several explanations have been offered [2], yet one widely accepted is that adipose tissue serves more than mere neighboring organ insulator and triglyceride depot storage [3]. In fact, adipocytes produce and secrete a variety or proteins, collectively termed adipokines that exhibit important metabolic and inflammatory properties, [3]. Of particular interest are adipokines leptin, adiponectin and visfatin, each with unique properties.

Leptin, the primary adipokine, has a primary role in appetite suppression and downregulation of food intake
$[2,3]$. Thus, knock-out mice are massively obese. In humans though only few cases have been described, while the common form of obesity is presented with high leptin levels, indicating a condition of leptin resistance. Insulin resistance and diabetes are also associated with higher leptin levels. Moreover, leptin has been found to exhibit a role in lipoprotein and glucocorticoid metabolism, as well as in immune function. Similarly to leptin, adiponectin is mainly produced from adipose tissue, as the most abundant adipokine [2, 3]. Adiponectin levels are decreased in obesity, insulin resistance, and type 2 diabetes. It has been proposed that low circulating adiponectin levels are indicative of increased cardiovascular risk, since it exhibited antiatherogenic, insulin-sensitizing, and anti-inflammatory properties. Visfatin, initially identified as a growth factor for early B-cells, is mainly produced by visceral fat $[4,5]$. It exerts insulin-mimetic effects in vivo and in vitro and is upregulated in obesity, metabolic syndrome, and diabetes. It is also implicated in dyslipidaemia, hypertension, and 
generally atherosclerotic-related diseases $[4,5]$. We have recently shown that subjects with metabolic abnormalities that predispose to the development of cardiovascular disease exhibit increased serum visfatin concentrations $[4,5]$. However, the pathophysiologic significance of this relationship remains to be elucidated.

Combating obesity has been under scientific investigation for many years, and several strategies are being developed to manage this public health issue. Dietary modulation is considered first-treatment option. Plant-derived polyphenols, such as flavonoids and resveratrol, as part of human nutrition, have been put on test. Epidemiological and clinical evidence suggests a protective role against cardiovascular disease [6-8]. In addition, results from both animal and clinical studies are promising regarding antiobesity effects [9-13]. In the present study, we aim to investigate the direct effect of flavonoids quercetin, epigallocatechin gallate (EGCG), and resveratrol on the secretion of leptin, adiponectin, and visfatin from human adipocytes in culture.

\section{Methods}

Quercetin, EGCG, resveratrol, Dulbecco's modified Eagle's medium (DMEM)/Ham's F12, penicillin/streptomycin, biotin, pantothenate, L-glutamine, isobutylmethylxanthine (IBMX), dexamethasone, insulin, transferring triiodothyronine (T3), were purchased from Sigma (St. Louis, USA), fetal calf serum (FCS) from GIBCO (California, USA), and rosiglitazone (BRL49653) from Cayman Chemicals (Mich, USA). Simpson-Golabi-Behmel syndrome (SGBS) cells were maintained and differentiated into adipocytes as previously described [14]. In brief, cells were cultured in 24-well plates in DMEM/Ham's F12 supplemented with 10\% FCS, penicillin/streptomycin, L-glutamine, $33 \mu \mathrm{M}$ biotin, and $17 \mu \mathrm{M}$ pantothenic acid and incubated at $37^{\circ} \mathrm{C}$ in a humidified atmosphere of $5 \% \mathrm{CO}_{2}$. Cells were grown to confluence for 5 days. Subsequently, we initiated the adipocyte differentiation program. Medium was changed into serumfree DMEM/F12, containing penicillin/streptomycin, biotin, pantothenic acid, $0.01 \mathrm{mg} / \mathrm{mL}$ transferrin, $0.1 \mu \mathrm{M}$ cortisol, $200 \mathrm{pM}$ T3, $20 \mathrm{nM}$ human insulin, $0.25 \mu \mathrm{M}$ dexamethasone, $500 \mu \mathrm{M}$ IBMX, and $2 \mu \mathrm{M}$ rosiglitazone (differentiation medium). On day 4, medium was replaced by the differentiation medium lacking rosiglitazone, IBMX, and dexamethasone (medium 2) and cells were cultured for additional 11 days in medium 2, which was changed every 3 days [14].

The cell differentiation into adipocytes was confirmed by (1) microscopic observation with simultaneous oil red O staining ( $0.5 \%$ oil red O) [15]; (2) determination of the triglyceride (TG) content in the cell lysate with the GPO Trinder method [16]. To prepare the cell-lysate, adipocytes were washed 3 times with phosphate-buffered saline (PBS, $\mathrm{pH}$ 7.4) and then were lysed with $200 \mu \mathrm{L}$ of lysis buffer containing $0.1 \%$ Triton X in PBS. Cell lysate was further homogenized by sonication. The protein content of cell lysate was determined by the Lowry method [17].

The effect of polyphenols on adipokine secretion was studied on fully differentiated adipocytes, that is, at 15 days [14]. Three independent experiments were performed in duplicate: SGBS adipocytes were rinsed twice with PBS and then treated for 24 and 48 hours with medium 2, containing additionally quercetin, EGCG, or resveratrol. All polyphenols were dissolved in dimethyl sulfoxide (DMSO). The maximum DMSO concentration into the culture medium did not exceed $0.2 \%$ by volume. Secreted adipokines were determined in duplicate in the cell supernatant collected from each well. Leptin and adiponectin were measured using kits purchased from BioVendor (Czech Republic) and visfatin with a kit from Alpco (Salem, USA) according to manufacturer's protocol. Coefficient of variation for within and between kits was less than $7 \%$ for all three kits. Each concentration obtained was determined from the standard curve and after adjustment for protein lysate content of respective well. Results are reported as means with the standard error of the mean. Statistical analysis was performed using SPPS statistical package for Windows (SPSS Inc., Chicago, Ill, USA). Distribution was evaluated by Kolmogorov-Smirnov test, and comparisons of only two group means were performed by Student's $t$-test. Significance was set at $P \leq 0.05$.

\section{Results}

We obtained fully differentiated mature adipocytes, 15 days after differentiation program induction, in accordance with previously published results [14]. Adipocyte differentiation was confirmed by microscopic observation of cells stained with oil red $\mathrm{O}$ (data not shown) and by quantitation of the cell TG content, expressed per $\mu \mathrm{g}$ of cell protein. Before the initiation of the differentiation program, the TG concentration was lower than the detection limit of the method used in the present study (i.e., lower than $16 \mu \mathrm{g} / \mathrm{mL}$ ); however, 15-days differentiated mature adipocytes contained detectable amounts of TG having a TG to cell protein ratio of $1.14 \pm 0.14 \mu \mathrm{g} / \mu \mathrm{g}$ (no difference was observed between various wells). Mature adipocytes secrete in the supernatant all three adipokines evaluated in the present study, 24 hours after changing the medium 2, whereas none of them was detected in the supernatant from cultured undifferentiated fibroblasts at day 4 (data not shown), thus further indicating functionality of adipocytes (leptin $98 \pm 22 \mathrm{ng} / \mathrm{mg}$ cell protein, adiponectin $81 \pm 23 \mathrm{ng} / \mathrm{mg}$ cell protein, visfatin $320 \pm$ $70 \mathrm{ng} / \mathrm{mg}$ cell protein).

The effect of polyphenols used in the present study on adipokine secretion was studied as described in Materials and Methods. In preliminary experiments, we used polyphenol concentrations ranging from 10 to $50 \mu \mathrm{M}$. However, at concentrations higher than $40 \mu \mathrm{M}$, a precipitate was observed in the cell supernatant for all polyphenols, immediately after the substance addition. Hence, we evaluated their effect on adipokines' secretion at lower concentrations, that is, at 10 and $25 \mu \mathrm{M}$. None of the three polyphenols tested influenced cell TG/protein ratio and leptin or adiponectin concentration in the cell supernatant at 24 hours and 48 hours (Figure 1). Similarly, EGCG had no effect on visfatin secretion at both time points. By contrast, incubation of adipocytes with quercetin resulted in a significant dose-dependent reduction of visfatin secretion (Figure 2). More specifically, quercetin 


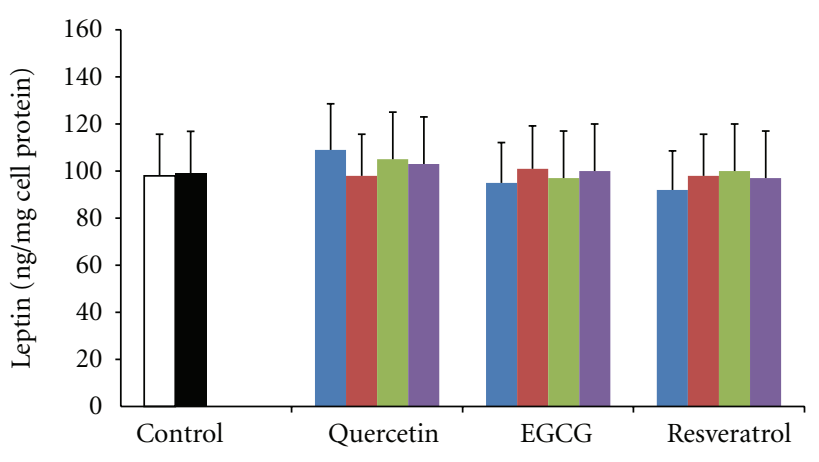

(a)

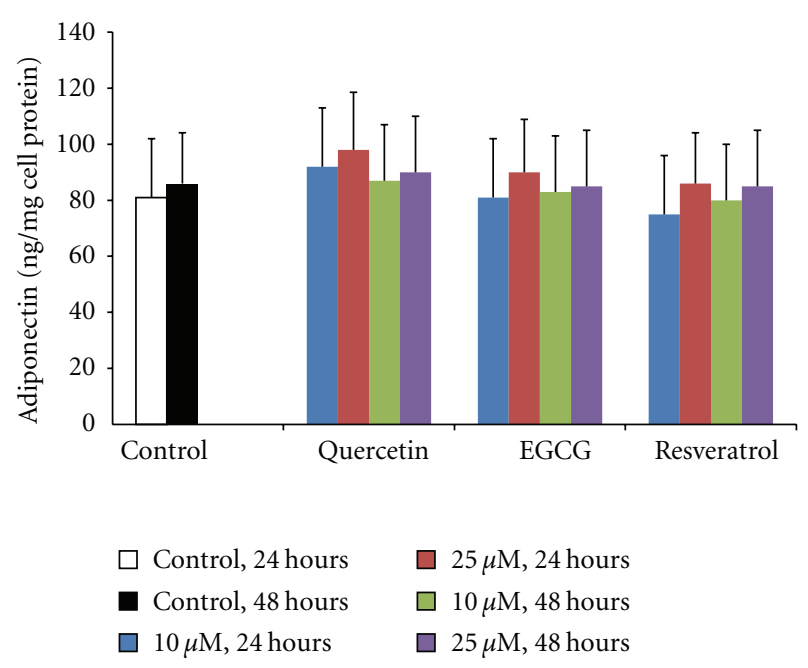

(b)

Figure 1: Polyphenols quercetin, epigallocatechin gallate (EGCG), and resveratrol do not affect leptin (a) and adiponectin (b) secretion by human mature SGBS adipocytes. Cells were cultured in medium and incubated with 10 or $25 \mu \mathrm{M}$ of quercetin, EGCG, and resveratrol for 24 and 48 hours. Leptin and adiponectin were determined in the cell supernatant by commercially available methods. Values represent the mean $\pm \mathrm{SD}$.

$10 \mu \mathrm{M}$ reduced visfatin secretion by $16 \%$ and $25 \%$ at 24 and 48 hours, respectively $(P<0.05$ versus control), whereas the corresponding changes for quercetin $25 \mu \mathrm{M}$ were $47 \%$ and $48 \%(P<0.05$ versus control and $P<0.05$ versus $10 \mu \mathrm{M}$ in the same time points). Low concentration of resveratrol had no effect on visfatin secretion, while the $25 \mu \mathrm{M}$ of the substance decreased visfatin concentration by $28 \%$ and $38 \%$ at 24 and 48 hours, respectively, $(P<0.05$ versus control for both comparisons). These changes were similar to those observed with the high dose of quercetin.

In order to evaluate whether the decrease in the visfatin concentration in the cell supernatant of cells treated with quercetin or resveratrol is due to the reduction in visfatin production, we determined the quercetin and resveratrol effects on both the cell-associated and the secreted visfatin levels. We measured intracellular and secreted visfatin levels in the presence of both polyphenols at both concentrations tested. Measuring intracellular visfatin in cell lysate yielded minimal amounts, in the order of hundredth relative to

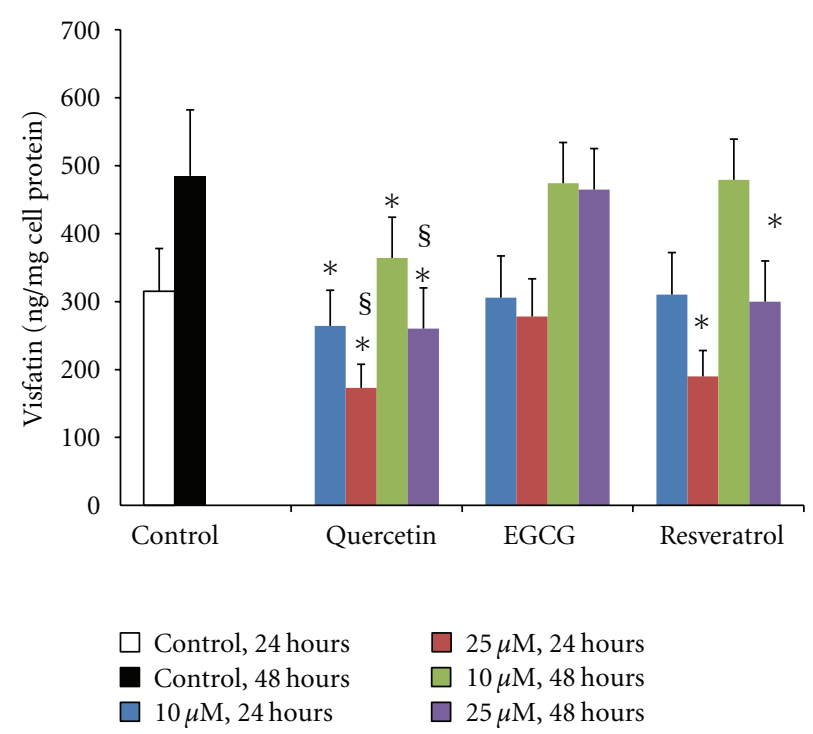

FIGURE 2: Effect of polyphenols quercetin, epigallocatechin gallate (EGCG), and resveratrol on visfatin secretion by human mature SGBS adipocytes at 24 and 48 hours. Cells were cultured in medium and incubated with 10 or $25 \mathrm{mM}$ of quercetin, EGCG, and resveratrol for 24 and 48 hours. Visfatin levels were determined in the cell supernatant by commercially available methods. Values represent the mean \pm SD. ${ }^{*} P<0.05$ compared with corresponding control, ${ }^{\S} P<0.05$ compared with the value obtained at $10 \mu \mathrm{M}$.

the total visfatin in the supernatant and without difference between wells $(4.1 \pm 0.9 \mathrm{ng}$ visfatin/mg cell protein, $\mathrm{p}=\mathrm{NS}$ between wells), suggesting that (1) in SGBS adipocytes, visfatin is a secretory protein and (2) quercetin and resveratrol reduced its production.

\section{Discussion}

Obesity is now considered a low-grade inflammatory state. Thus, it is supported that its management should not solely be targeted to weight loss, but also towards correction of metabolic imbalance of adipokines. In the present study, we aimed to investigate possible effect of three specific plant polyphenols on adipokine secretion from human adipocytes in culture. We selected three polyphenols which have been considered representative, more abundant in human nutrition and more promising in terms of positive effects in previous studies $[18,19]$. Several investigators have evaluated the role of various flavonoids or resveratrol concerning their effects on preadipocyte differentiation, adipogenesis, and adipocyte apoptosis [20]. We focused on a different aspect of adipocyte metabolism, adipokine secretion. The usual model selected in previous studies is mouse strain 3T3-L1 or isolated rat adipocytes. However, in this study, we preferred to use the human SGBS cell line, because, given the species differences in adipose tissue metabolism [21], this cell line may more closely resemble human adipose tissue.

To the best of our knowledge, this is the first report on the polyphenol-induced modulation of visfatin secretion by human SGBS adipocytes. Interestingly, measuring visfatin 
in the lysate yielded minimal amounts, suggesting that the majority of total visfatin is excreted in the medium, in accordance with the findings of Revollo et al. in SGBS adipocytes [22]. Thus, we presume that polyphenols exert their effects on visfatin metabolism by modulating both the production and excretion of this molecule by SGBS adipocytes. In addition, our results support a substancedependent differential effect of various polyphenols on visfatin secretion, a finding that may be related to the chemical structure of the polyphenols tested. Indeed, we found that quercetin more potently reduced visfatin secretion compared to resverartol (although these differences did not reach statistical significance), while the structurally different EGCG did not change visfatin secretion. Similarly, Chuang et al. [23] have recently demonstrated that quercetin more efficiently improves anti-inflammatory pathways and insulin resistance than resveratrol. Differential effects of different flavonoids on various aspects of adipose tissue metabolism have been reported elsewhere as well [24, 25]. Visfatin levels have been found increased in obesity, diabetes mellitus, hypertension, and cardiovascular disease. Although the nature of this relationship is still under investigation, the evidence points towards a proatherogenic role of this adipokine. In this regard, in vitro visfatin downregulation by polyphenols might be related to their antiatherogenic properties.

The effect of polyphenols on leptin metabolism remains controversial. Thus, Tsuda et al. [26] showed that anthocyanin, another flavonoid, induced an increase in leptin levels in cell medium of isolated rat adipocytes. However, in our study, none of the polyphenols tested had an effect on leptin secretion. These differences can, at least in part, be attributed to that, by contrast to Tsuda et al., we did not incubate the cells with an inflammatory stimulant, such as TNF, that may increase baseline leptin levels. To make things more complicate, studies on rodents fed a diet rich in polyphenols or injected with EGCG revealed no change or even a decrease in serum leptin levels [27-30]. Certainly, comparison between cell cultures and animal studies is not direct.

We showed no change on adiponectin secretion in the medium with either treatment, a finding which suggests that, at least in mature adipocytes, the polyphenols studied have no effect. Interestingly, though, anthocyanin was reported to increase adiponectin gene expression and protein secretion in cultured rat adipocytes and tissue gene expression experiments [26]. However, apart from using different flavonoids than ours, the concentration used in this study was higher (4 to 10 fold). Yet, in a recent study by Cho et al. [25], some flavonoids, including quercetin and EGCG, decreased adiponectin in mouse 3T3-L1 adipocytes, whereas others increased it, thus further strengthening the view that various flavonoids may possess diverse effects and that adipose tissue metabolism may display important interspecies differences. In fact, even within the same model (3T3-L1) and between in vitro and in vivo studies, results can be contrasting $[28,31]$. In human visceral adipocytes from obese women, resveratrol increased adiponectin [32]. Maybe, cultured SGBS adipocytes that are not treated with a proinflammatory stimulant exhibit a higher basal adiponectin excretion than (theoretically) more inflamed visceral adipocytes that may counteract inflammation after treatment with resveratrol. In addition, whole body metabolism of an antioxidant mix or green tea infusion including EGCG may impact indirectly on adipose tissue and adiponectin, for example, via improved liver function [33].

In conclusion, from the polyphenols studied, quercetin and resveratrol decreased visfatin secretion. No effect of the tested polyphenols on leptin and adiponectin secretion from human adipocytes in culture was noticed. To the best of our knowledge, this is the first time polyphenols are reported to modify visfatin secretion from cultured adipocytes. This finding might be a potentially beneficial effect of these compounds on adipose tissue and could contribute to their antiatherogenic actions. In the future, these polyphenols might be used pharmacologically for treating obesity-related metabolic abnormalities. Further research is required to verify our findings in cell cultures as well as in animal and human studies.

\section{References}

[1] P. G. Kopelman, "Obesity as a medical problem," Nature, vol. 404, no. 6778, pp. 635-643, 2000.

[2] L. F. Van Gaal, I. L. Mertens, and C. E. De Block, "Mechanisms linking obesity with cardiovascular disease," Nature, vol. 444, no. 7121, pp. 875-880, 2006.

[3] D. C. W. Lau, B. Dhillon, H. Yan, P. E. Szmitko, and S. Verma, "Adipokines: molecular links between obesity and atheroslcerosis," The American Journal of Physiology, vol. 288, no. 5, pp. H2031-H2041, 2005.

[4] T. D. Filippatos, C. S. Derdemezis, D. N. Kiortsis, A. D. Tselepis, and M. S. Elisaf, "Increased plasma levels of visfatin/pre$\mathrm{B}$ cell colony-enhancing factor in obese and overweight patients with metabolic syndrome," Journal of Endocrinological Investigation, vol. 30, no. 4, pp. 323-326, 2007.

[5] T. D. Filippatos, H. S. Randeva, C. S. Derdemezis, M. S. Elisaf, and D. P. Mikhailidis, "Visfatin/PBEF and atherosclerosisrelated diseases," Current Vascular Pharmacology, vol. 8, no. 1, pp. 12-28, 2010.

[6] M. G. L. Hertog, D. Kromhout, C. Aravanis et al., "Flavonoid intake and long-term risk of coronary heart disease and cancer in the seven countries study," Archives of Internal Medicine, vol. 155, no. 4, pp. 381-386, 1995.

[7] M. Monagas, N. Khan, C. Andres-Lacueva et al., "Effect of cocoa powder on the modulation of inflammatory biomarkers in patients at high risk of cardiovascular disease," The American Journal of Clinical Nutrition, vol. 90, no. 5, pp. 11441150, 2009.

[8] L. A. E. Hughes, I. C. W. Arts, T. Ambergen et al., "Higher dietary flavone, flavonol, and catechin intakes are associated with less of an increase in BMI over time in women: a longitudinal analysis from the Netherlands Cohort Study," The American Journal of Clinical Nutrition, vol. 88, no. 5, pp. 13411352, 2008.

[9] M. Bose, J. D. Lambert, J. Ju, K. R. Reuhl, S. A. Shapses, and C. S. Yang, "The major green tea polyphenol, (-)epigallocatechin-3-gallate, inhibits obesity, metabolic syndrome, and fatty liver disease in high-fat-fed mice," The Journal of Nutrition, vol. 138, no. 9, pp. 1677-1683, 2008. 
[10] L. K. Stewart, J. L. Soileau, D. Ribnicky et al., "Quercetin transiently increases energy expenditure but persistently decreases circulating markers of inflammation in C57BL/6J mice fed a high-fat diet," Metabolism, vol. 57, no. 1, pp. S39-S46, 2008.

[11] L. Rivera, R. Moron, M. Sanchez, A. Zarzuelo, and M. Galisteo, "Quercetin ameliorates metabolic syndrome and improves the inflammatory status in obese Zucker rats," Obesity, vol. 16, no. 9, pp. 2081-2087, 2008.

[12] A. Basu, K. Sanchez, M. J. Leyva et al., "Green tea supplementation affects body weight, lipids, and lipid peroxidation in obese subjects with metabolic syndrome," Journal of the American College of Nutrition, vol. 29, no. 1, pp. 31-40, 2010.

[13] H. Wang, Y. Wen, Y. Du et al., "Effects of catechin enriched green tea on body composition," Obesity, vol. 18, no. 4, pp. 773-779, 2010.

[14] M. Wabitsch, R. E. Brenner, I. Melzner et al., "Characterization of a human preadipocyte cell strain with high capacity for adipose differentiation," International Journal of Obesity, vol. 25 , no. 1, pp. 8-15, 2001.

[15] J. L. Ramirez-Zacarias, F. Castro-Munozledo, and W. KuriHarcuch, "Quantitation of adipose conversion and triglycerides by staining intracytoplasmic lipids with oil red O," Histochemistry, vol. 97, no. 6, pp. 493-497, 1992.

[16] P. Fossati and L. Prencipe, "Serum triglycerides determined colorimetrically with an enzyme that produces hydrogen peroxide," Clinical Chemistry, vol. 28, no. 10, pp. 2077-2080, 1982.

[17] O. H. Lowry, N. J. Rosebrough, A. L. Farr, and R. J. Randall, "Protein measurement with the Folin phenol reagent," The Journal of Biological Chemistry, vol. 193, no. 1, pp. 265-275, 1951.

[18] K. A. Grove and J. D. Lambert, "Laboratory, epidemiological, and human intervention studies show that tea (Camellia sinensis) may be useful in the prevention of obesity," The Journal of Nutrition, vol. 140, no. 3, pp. 446-453, 2010.

[19] V. R. Ramprasath and P. J. H. Jones, "Anti-atherogenic effects of resveratrol," European Journal of Clinical Nutrition, vol. 64, no. 7, pp. 660-668, 2010.

[20] C. L. Hsu and G. C. Yen, "Phenolic compounds: evidence for inhibitory effects against obesity and their underlying molecular signaling mechanisms," Molecular Nutrition and Food Research, vol. 52, no. 1, pp. 53-61, 2008.

[21] P. Wang, E. Mariman, J. Renes, and J. Keijer, "The secretory function of adipocytes in the physiology of white adipose tissue," Journal of Cellular Physiology, vol. 216, no. 1, pp. 3-13, 2008.

[22] J. R. Revollo, A. Korner, K. F. Mills et al., "Nampt/PBEF/ visfatin regulates insulin secretion in $\beta$ cells as a systemic NAD biosynthetic enzyme," Cell Metabolism, vol. 6, no. 5, pp. 363$375,2007$.

[23] C. C. Chuang, K. Martinez, G. Xie et al., "Quercetin is equally or more effective than resveratrol in attenuating tumor necrosis factor- $\alpha$-mediated inflammation and insulin resistance in primary human adipocytes," The American Journal of Clinical Nutrition, vol. 92, no. 6, pp. 1511-1521, 2010.

[24] A. W. Harmon and J. B. Harp, "Differential effects of flavonoids on 3T3-L1 adipogenesis and lipolysis," The American Journal of Physiology, vol. 280, no. 4, pp. C807-C813, 2001.

[25] S. Y. Cho, P. J. Park, H. J. Shin et al., “(-)-catechin suppresses expression of Kruppel-like factor 7 and increases expression and secretion of adiponectin protein in 3T3-L1 cells," The American Journal of Physiology, vol. 292, no. 4, pp. E1166E1172, 2007.
[26] T. Tsuda, Y. Ueno, H. Aoki et al., "Anthocyanin enhances adipocytokine secretion and adipocyte-specific gene expression in isolated rat adipocytes," Biochemical and Biophysical Research Communications, vol. 316, no. 1, pp. 149-157, 2004.

[27] N. Chen, R. Bezzina, E. Hinch et al., "Green tea, black tea, and epigallocatechin modify body composition, improve glucose tolerance, and differentially alter metabolic gene expression in rats fed a high-fat diet," Nutrition Research, vol. 29, no. 11, pp. 784-793, 2009.

[28] S. Wein, N. Behm, R. K. Petersen, K. Kristiansen, and S. Wolffram, "Quercetin enhances adiponectin secretion by a PPAR- $\gamma$ independent mechanism," European Journal of Pharmaceutical Sciences, vol. 41, no. 1, pp. 16-22, 2010.

[29] K. Décordé, P. L. Teissèdre, T. Sutra, E. Ventura, J. P. Cristol, and J. M. Rouanet, "Chardonnay grape seed procyanidin extract supplementation prevents high-fat diet-induced obesity in hamsters by improving adipokine imbalance and oxidative stress markers," Molecular Nutrition and Food Research, vol. 53, no. 5, pp. 659-666, 2009.

[30] Y. H. Kao, R. A. Hiipakka, and S. Liao, "Modulation of endocrine systems and food intake by green tea epigallocatechin gallate," Endocrinology, vol. 141, no. 3, pp. 980-987, 2000.

[31] M. Shimada, K. Mochizuki, N. Sakurai, and T. Goda, "Dietary supplementation with epigallocatechin gallate elevates levels of circulating adiponectin in non-obese type-2 diabetic GotoKakizaki rats," Bioscience, Biotechnology and Biochemistry, vol. 71, no. 8, pp. 2079-2082, 2007.

[32] C. S. Costa, F. Rohden, T. O. Hammes et al., "Resveratrol upregulated SIRT1, FOXO1, and adiponectin and downregulated PPAR $\gamma 1-3$ mRNA expression in human visceral adipocytes," Obesity Surgery, vol. 21, no. 3, pp. 356-361, 2010.

[33] G. C. Bakker, M. J. van Erk, L. Pellis et al., "An antiinflammatory dietary mix modulates inflammation and oxidative and metabolic stress in overweight men: a nutrigenomics approach," The American Journal of Clinical Nutrition, vol. 91, no. 4, pp. 1044-1059, 2010. 

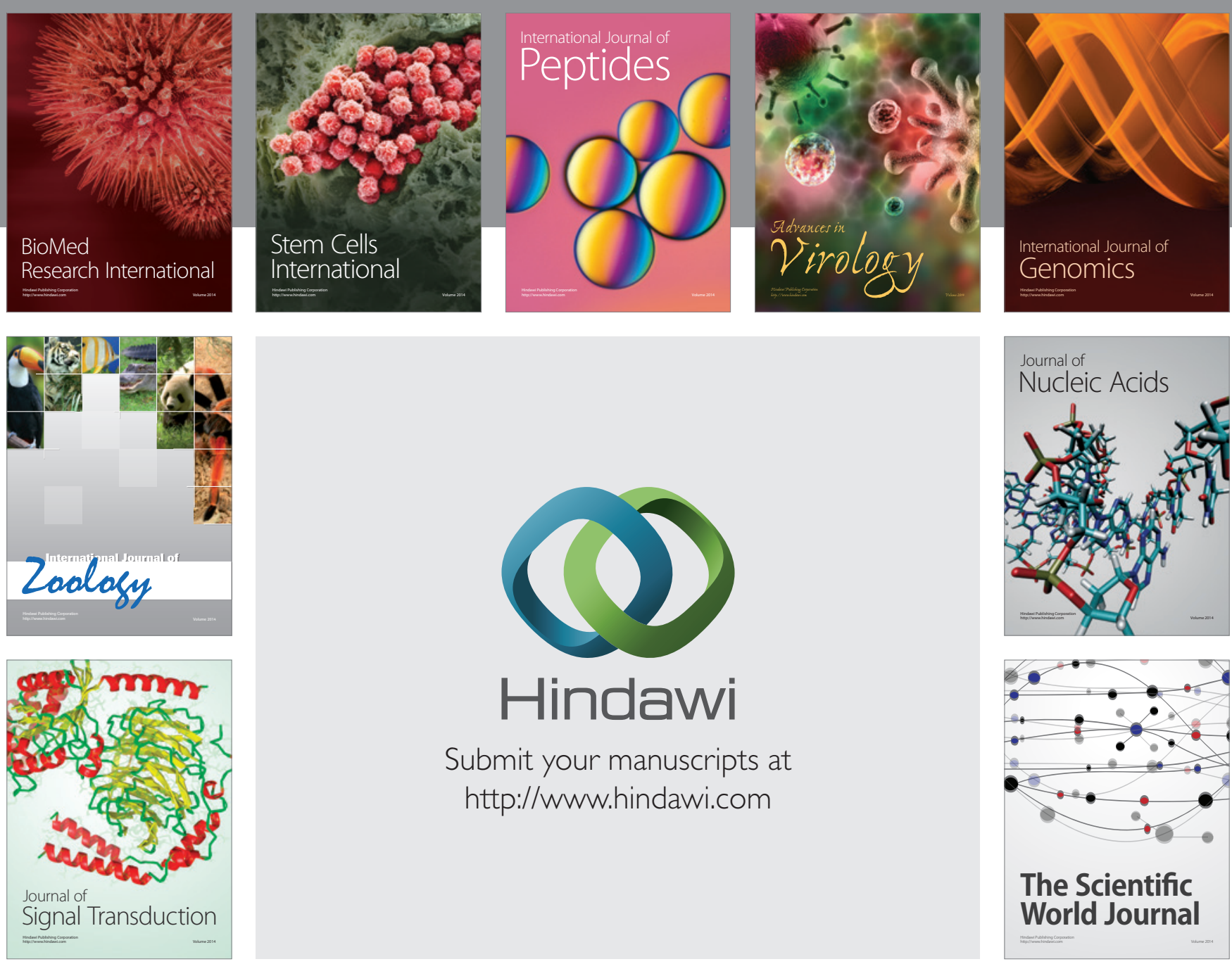

Submit your manuscripts at

http://www.hindawi.com
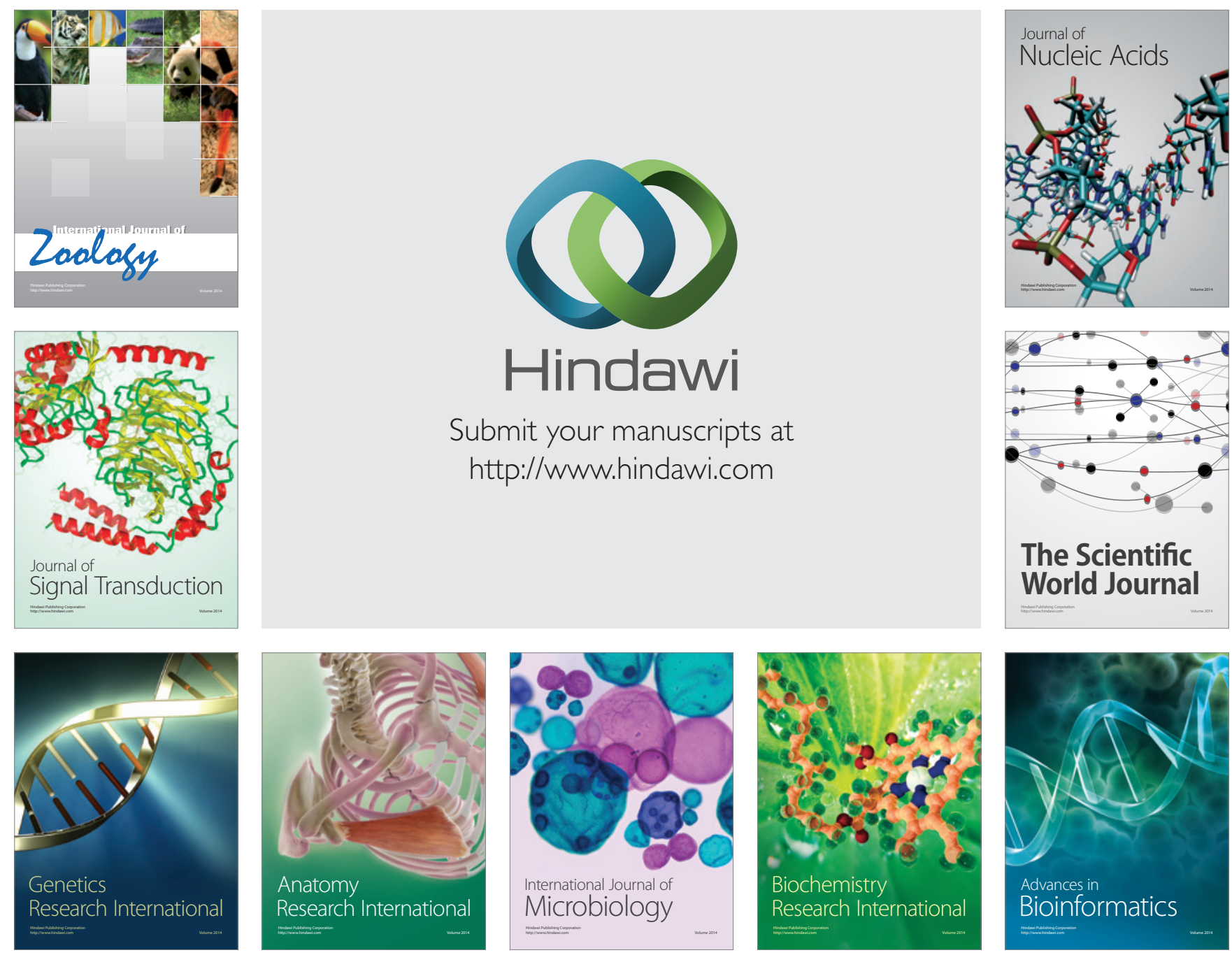

The Scientific World Journal
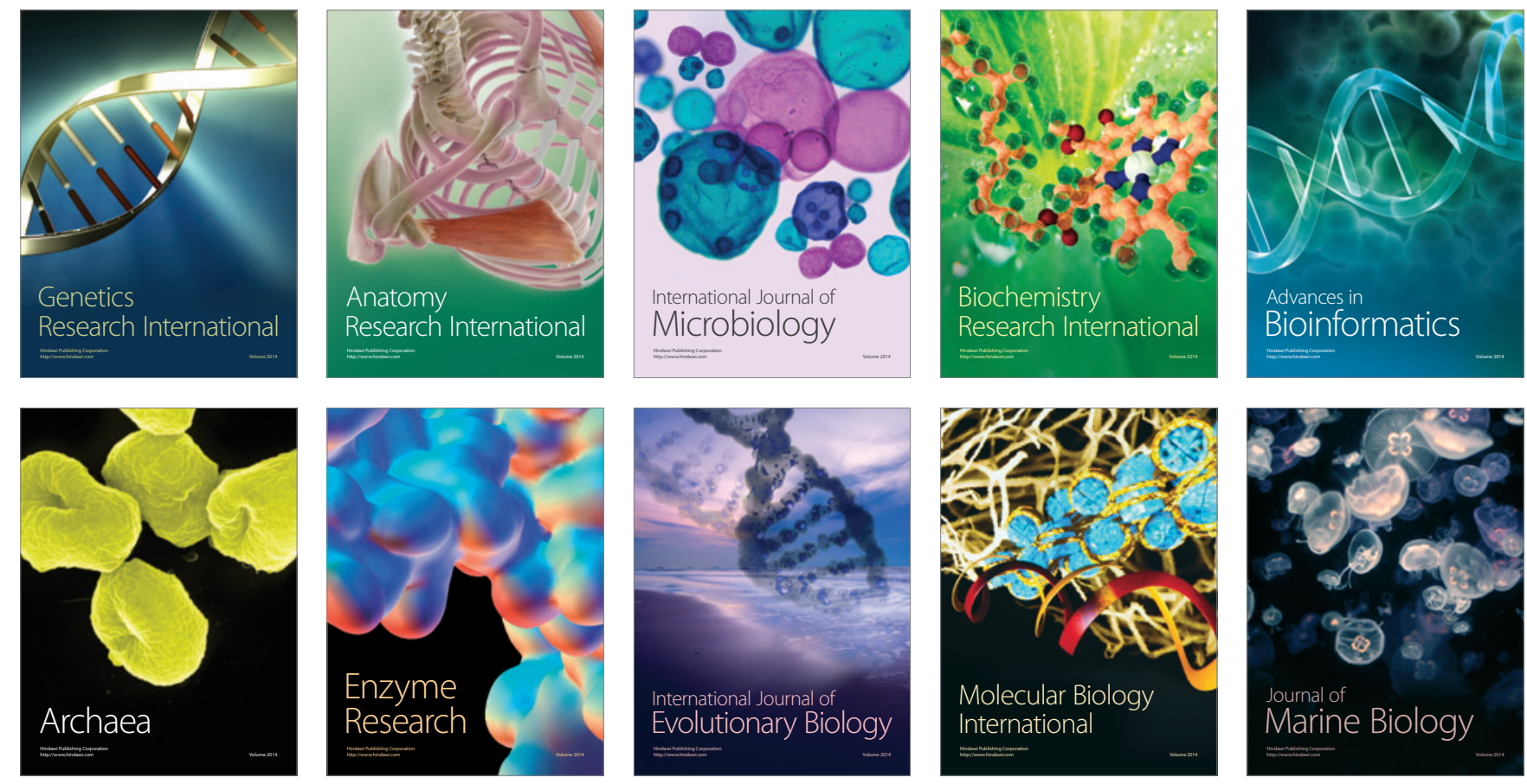